University of Nebraska - Lincoln DigitalCommons@University of Nebraska - Lincoln

CSE Conference and Workshop Papers

Computer Science and Engineering, Department of

2010

\title{
Vision Graph Construction in Wireless Multimedia Sensor Networks
}

Xin Dong

University of Nebraska-Lincoln, xdong@cse.unl.edu

Mehmet C. Vuran

University of Nebraska-Lincoln, mcvuran@cse.unl.edu

Follow this and additional works at: http://digitalcommons.unl.edu/cseconfwork

Dong, Xin and Vuran, Mehmet C., "Vision Graph Construction in Wireless Multimedia Sensor Networks" (2010). CSE Conference and Workshop Papers. 285.

http://digitalcommons.unl.edu/cseconfwork/285

This Article is brought to you for free and open access by the Computer Science and Engineering, Department of at DigitalCommons@University of Nebraska - Lincoln. It has been accepted for inclusion in CSE Conference and Workshop Papers by an authorized administrator of

DigitalCommons@University of Nebraska - Lincoln. 


\title{
Vision Graph Construction in Wireless Multimedia Sensor Networks
}

\author{
Xin Dong and Mehmet C. Vuran \\ Cyber-Physical Networking Laboratory \\ Department of Computer Science \& Engineering \\ University of Nebraska-Lincoln, Lincoln, NE 68588 \\ Email: \{xdong, mcvuran\}@cse.unl.edu
}

\begin{abstract}
In Wireless multimedia sensor networks (WMSNs), the camera nodes connected in the vision graph share overlapped field of views (FOVs) and they depend on the densely deployed relay nodes in the communication network graph to communicate with each other. Given a uniformly deployed camera sensor network with relay nodes, the problem is to find the number of hops for the vision-graph-neighbor-searching messages to construct the vision graph in an energy efficient way. In this paper, mathematical models are developed to analyze the FOV overlap of the camera nodes and the multi-hop communications in two dimensional topologies, which are utilized to analyze the relation between vision graph construction and maximum hop count. In addition, simulations are conducted to verify the models.
\end{abstract}

\section{INTRODUCTION}

Recently, the advances in low cost CMOS imaging sensors have made wireless multimedia sensor networks (WMSNs) possible [1][5]. In WMSNs, multiple camera sensors are deployed to monitor an area. Potential applications of WMSNs include surveillance, traffic tracking, wildlife monitoring, and battlefield monitoring.

The challenges faced by scalar wireless sensor networks, such as energy constraints, limited processing capabilities, unreliability and low accuracy of obtained data, are only exacerbated in WMSNs. To improve the sensing quality, a certain level of collaboration among sensor nodes are required. In [1], collaborative multimedia in-network processing is suggested, which can utilize the computational capacity of sensors as well as reduce communication cost and energy consumption. In [2], scalar sensors are exploited to help camera nodes detect events in WMSNs. More directly, camera sensors can collaborate with each other to accomplish tasks. This is achieved by exploiting the overlapped field-of-views of the camera sensors.

In a WMSN, each camera sensor has its own directional sensing range, known as the field-of-view (FOV), and cameras may have overlapped FOVs such that if an event occurs in this overlapped area, several cameras may capture this event in different perspectives. These camera nodes form a vision graph [4], in which an edge between two cameras indicates they share an overlapped FOV and the two cameras are called vision graph neighbors. Some research exploits the characteristics of the overlapped FOVs in collaboration of camera sensor nodes. In [6], routing paths are established based on the overlapped FOVs of camera nodes. In [3] and [8], overlapped FOVs are exploited to define correlation among camera nodes and this correlation is used for cooperative video processing. Meanwhile, in [4], the images of the overlapped FOVs have been explored to calibrate the camera nodes.

To construct vision graphs in WMSNs, two methods are widely used in literature. The cameras nodes may know their locations and directions a priori [3], or reference objects are used for camera nodes to calculate overlapped FOVs [7][8]. However, in these works, simple flooding is employed to exchange information among camera nodes. It is well known that for large scale WSNs, flooding is impractical in terms of communication cost and network congestion. In this paper, we propose a limited-hop-number multi-hop communication for constructing the vision graph in WMSNs. Given a uniformly deployed camera sensor network with relay nodes, we are interested in finding the maximum allowed hop count for the vision-graph-neighbor-searching messages (hello message) in order to construct the vision graph in an energy efficient way.

Mathematical models are developed to analyze the probability of constructing the vision graph for different message lifetime (maximum hop count). This is achieved by developing a probabilistic model of the overlapped FOV for different distance and the probabilistic model of the propagation distance for different message life time. In [9], the authors have proposed a method to analyze the probability distribution of multi-hop communications in WSNs. However, only one dimensional topology is considered and the channel is assumed to be perfect. In this paper, we propose a recursive method to analyze the multi-hop communications of WMSNs in two dimensional topologies. Moreover, a more practical channel model is adopted to capture the feature of wireless communication. In addition, simulations are conducted to verify our models.

To the best of our knowledge, this is the first paper to address the communication problem of constructing the vision graph in WMSNs. Our main contributions are:

- The probabilistic model of two cameras having an overlapped FOV with respect to their distance;

- The 2-D multi-hop communication model that maps maximum hop number to connectivity;

- The mathematical model to calculate the needed maximum hop number to construct the vision graph for a given WMSN.

The remainder of this paper is organized as follows: The probabilistic models for the overlapped FOV, the multi-hop communication and the vision graph construction are developed in Section II. Simulations and Numerical analysis of those models are provided in Section III. Furthermore, the conclusions are 


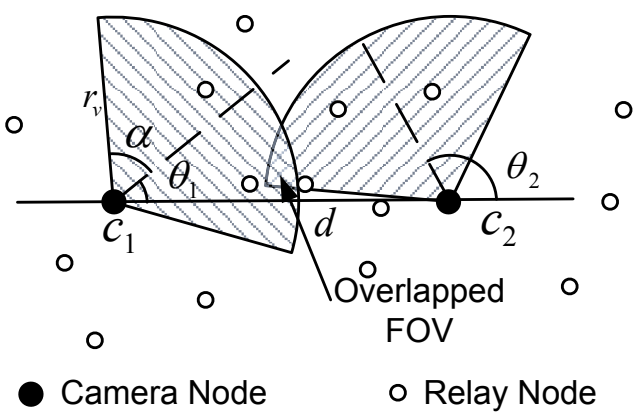

Fig. 1. The system overview.

drawn in Section IV.

\section{SySTEM MODEL}

We consider applications in which the camera sensors are randomly deployed. To connect these camera sensors, other relay nodes are also randomly deployed among them by Poisson random process. After deployment, the camera sensors send hello messages that have a maximum allowed hop count to find their vision graph neighbors. In each transmission, the sender broadcast a hello message unless the message achieves its maximum allowed hop count. When a camera sensor receives a hello message from its peers, it uses the location and direction information to calculate if the source of the message is its vision graph neighbor. Hence the vision graph is constructed in a distributed manner.

Denote the probability of constructing the vision graph $p_{v}$, our goal is to relate this probability to the maximum allowed hop count, $n$, of the hello message. $p_{v}$ is given as

$$
p_{v}(n)=\frac{\int_{0}^{\infty} p_{v c}(d, n) \mathrm{d} d}{\int_{0}^{\infty} p_{v}(d) \mathrm{d} d}
$$

where $p_{v c}(d, n)$ is the probability of successfully connected vision graph neighbors given the distance $d$ and the maximum hop number $n$ whereas $p_{v}(d)$ is the probability of having vision graph neighbors given distance $d . p_{v}(d)$ is related to the FOV of the camera and it is developed in Section II-A, while $p_{v c}(d, n)$ is related to the multi-hop communication of the sensors, and the model is provided in Section II-B. In Section II-C, those two models are utilized to develop the model for vision graph construction.

\section{A. Overlapped Field of View}

The FOV of a camera is modeled as a fan sector in a $2 \mathrm{D}$ plane, as shown in Fig. 1. It is defined by a tuple $\left(r_{v}, \alpha\right)$, where $r_{v}$ is the FOV radius, which determines the maximum distance the camera can observe, and $\alpha$ is the visual angle, which depicts the width of the FOV.

The overlap model is shown in Fig. 1. It is observed that given the distance $d$ of two cameras, when these two cameras are at some specific directions, they will have overlapped FOV and become vision graph neighbors. Given the assumption that the direction of the camera is uniformly distributed, the probability that two cameras are vision graph neighbors is equal to the portion of directions at which they have an overlapped
FOV. When given the direction of the first camera at angle $\theta_{1}$, there exists a range $\left[\theta_{2}^{\min }, \theta_{2}^{\max }\right]$, such that when the direction of the second camera, $\theta_{2}$, is in this range, these two cameras have an overlapped FOV. As $\theta_{1}$ changes, the range of $\theta_{2}$ changes too. With the ranges of $\theta_{1}$ and $\theta_{2}$ in which the two camera nodes can have an overlapped FOV at distance $d$, the probability of having an overlapped FOV at distance $d$, which is denoted as $p_{o}(d)$, is the integral of the ranges divided by the maximum range. Thus,

$$
p_{o}(d)=\frac{1}{2 \pi^{2}} \sum_{i} \int_{\theta_{1}^{\min , i}}^{\theta_{1}^{\max , i}}\left(\theta_{2}^{\max , i}-\theta_{2}^{\min , i}\right) \mathrm{d} \theta_{1} .
$$

In Table I, the ranges of $\theta_{1}$ and $\theta_{2}$, in which two camera nodes have an overlapped FOV for different distance, $d$, are listed. For a given $d$, the range of $\theta_{1}$ is divided into categories, and in each category, the range of $\theta_{2}$ can be expressed by the value of $\theta_{1}$. Note in the tables, the range of $\theta_{1}$ is only considered as in range $[0, \pi]$. For the range $[\pi, 2 \pi]$, it is a mirror of $[0, \pi]$. Also note that the camera visual angle $\alpha$ impacts the boundary conditions in (2), as shown in Table I and in three different cases $\left(\left[0, \frac{\pi}{4}\right],\left[\frac{\pi}{4}, \frac{\pi}{3}\right],\left[\frac{\pi}{3}, \frac{\pi}{2}\right]\right)$, the expressions are different. Here, we assume the visual angle of the camera $\alpha$ is in the range of $\left[\frac{\pi}{4}, \frac{\pi}{3}\right]$. The variables $a_{1}-a_{6}$ in Table $\mathrm{I}$ are given as follows:

$$
\begin{aligned}
& a_{1}=\cos ^{-1} \frac{d}{2 r_{v}} \\
& a_{2}=\tan ^{-1} \frac{r_{v} \sin \left(\theta^{-}\right)}{d-r_{v} \cos \left(\theta^{-}\right)} \\
& a_{3}=\sin ^{-1} \frac{r_{v}}{d} \\
& a_{4}=\cos ^{-1} \frac{d \tan ^{2}\left(\theta^{-}\right)+\sqrt{r_{v}^{2}+r_{v}^{2} \tan ^{2}\left(\theta^{-}\right)-d^{2} \tan ^{2}\left(\theta^{-}\right)}}{r_{v}\left(1+\tan ^{2}\left(\theta^{-}\right)\right)}
\end{aligned}
$$

$a_{5}=\cos ^{-1} \frac{d \tan ^{2}\left(\theta^{-}\right)-\sqrt{r_{v}^{2}+r_{v}^{2} \tan ^{2}\left(\theta^{-}\right)-d^{2} \tan ^{2}\left(\theta^{-}\right)}}{r_{v}\left(1+\tan ^{2}\left(\theta^{-}\right)\right)}$,

$a_{6}=\cos ^{-1} \frac{d \tan ^{2}\left(\theta^{+}\right)+\sqrt{r_{v}^{2}+r_{v}^{2} \tan ^{2}\left(\theta^{+}\right)-d^{2} \tan ^{2}\left(\theta^{+}\right)}}{r_{v}\left(1+\tan ^{2}\left(\theta^{+}\right)\right)}$,

where $\theta^{-}=\theta_{1}-\alpha$ and $\theta^{+}=\theta_{1}+\alpha$.

As expected, it is noticed from Table I that when the two cameras are close to each other, the ranges in which they can be vision graph neighbors are larger, which means the probability that they are vision graph neighbors is greater.

\section{B. Multi-hop Communication}

In this section, we develop a model to analyze multihop communication of two camera nodes in wireless sensor networks. In the analysis, the distance of camera node $A$ and $B$ is denoted as $l$ and the density of the relay nodes is $\lambda$. In addition, log-normal shadowing model is employed to represent the channel.

In our model, $C_{d}^{n}$ represents the event that two nodes at distance $d$ can communicate through exactly $n$ hops, while $\bar{C}_{d}^{n}$ represents the opposite. The probability of event $C_{d}^{n}$ is 
TABLE I

THE POSSIBLE RANGES OF $\theta_{1}$ AND $\theta_{2}$ TO HAVE AN OVERLAPPED FOV FOR DIFFERENT DISTANCE $d$.

\begin{tabular}{|c|c|c|}
\hline \multicolumn{3}{|c|}{$\sqrt{2} r_{v}<d \leq 2 r_{v}$} \\
\hline$i$ & $\theta_{1} \in\left[\theta_{1}^{\min , i}, \psi_{1}^{\max , i}\right]$ & $\theta_{2} \in\left[\theta_{2}^{\min , i}, \theta_{2}^{\max , i}\right]$ \\
\hline 1 & {$\left[0, \alpha-\cos ^{-1} \frac{d}{2 r_{v}}\right]$} & {$\left[\pi-a_{1}-\alpha, \pi+a_{1}+\alpha\right]$} \\
\hline 2 & {$\left[\alpha-\cos ^{-1} \frac{d}{2 r_{u}}, \alpha\right]$} & {$\left[\pi-a_{1}-\alpha, \pi-a_{2}+\alpha\right]$} \\
\hline 3 & {$\left[\alpha, \cos ^{-1} \frac{d}{2 r_{v}}+\alpha\right]$} & {$\left[\pi-a_{1}-\alpha, \pi-a_{4}+\alpha\right]$} \\
\hline \multicolumn{3}{|c|}{$r_{v}<d \leq \sqrt{2} r_{v}$} \\
\hline$i$ & $\theta_{1} \in\left[\theta_{1}^{\min , i}, \psi_{1}^{\max , i}\right]$ & $\theta_{2} \in\left[\theta_{2}^{\min , i}, \theta_{2}^{\max , i}\right]$ \\
\hline 1 & {$\left[0, \alpha-\cos ^{-1} \frac{r_{v}}{d}\right]$} & {$\left[-a_{3}+\pi-\alpha, a_{3}+\pi+\alpha\right]$} \\
\hline 2 & {$\left[\alpha-\cos ^{-1} \frac{r_{v}}{d}, \alpha\right]$} & {$\left[-a_{3}+\pi-\alpha, \pi-a_{2}+\alpha\right]$} \\
\hline 3 & {$\left[\alpha, \cos ^{-1} \frac{r_{v}}{d}+\alpha\right]$} & {$\left[-a_{3}+\pi-\alpha, \pi-a_{4}+\alpha\right]$} \\
\hline 4 & {$\left[\cos ^{-1} \frac{r_{v}}{d}+\alpha, \cos ^{-1} \frac{d}{2 r_{v}}+\alpha\right]$} & {$\left[\pi-a_{2}-\alpha, \pi-a_{4}+\alpha\right]$} \\
\hline 5 & {$\left[\cos ^{-1} \frac{d}{2 r_{v}}+\alpha, \sin ^{-1} \frac{r_{v}}{d}+\alpha\right]$} & {$\left[\pi-a_{5}-\alpha, \pi-a_{4}+\alpha\right]$} \\
\hline \multicolumn{3}{|c|}{$-2 r_{v} \cos (2 \alpha)<d \leq r$} \\
\hline$i$ & $\theta_{1} \in\left[\theta_{1}^{\min , i}, \psi_{1}^{\max , i}\right]$ & $\theta_{2} \in\left[\theta_{2}^{\min , i}, \theta_{2}^{\max , i}\right]$ \\
\hline 1 & {$[0, \alpha]$} & {$[0,2 \pi]$} \\
\hline 2 & {$\left[\alpha, \cos ^{-1} \frac{d}{2 r_{v}}+\alpha\right]$} & {$\left[\pi+a_{2}-\alpha, \pi+\alpha\right]$} \\
\hline 3 & {$\left[\cos ^{-1} \frac{d}{2 r_{v}}+\alpha, \pi-\alpha\right]$} & {$\left[\pi-a_{5}-\alpha, \pi+\alpha\right]$} \\
\hline 4 & {$\left[\pi-\alpha, \alpha+\frac{\pi}{2}\right]$} & {$\left[\pi-a_{5}-\alpha, a_{6}+\pi+\alpha\right]$} \\
\hline 5 & {$\left[\alpha+\frac{\pi}{2}, \pi\right]$} & {$\left[\pi-a_{4}-\alpha, a_{6}+\pi+\alpha\right]$} \\
\hline \multicolumn{3}{|c|}{$0<d \leq-2 r_{v} \cos (2 \alpha)$} \\
\hline$i$ & $\theta_{1} \in\left[\theta_{1}^{\min , i}, \psi_{1}^{\max , i}\right]$ & $\theta_{2} \in\left[\theta_{2}^{\min , i}, \theta_{2}^{\max , i}\right]$ \\
\hline 1 & {$[0, \alpha]$} & {$[0,2 \pi]$} \\
\hline 2 & {$[\alpha, \pi-\alpha]$} & {$\left[\pi-a_{2}-\alpha, \pi+\alpha\right]$} \\
\hline 3 & {$\left[\pi-\alpha, \cos ^{-1} \frac{d}{2 r_{v}}+\alpha\right]$} & {$\left[\pi-a_{2}-\alpha, a_{6}+\pi+\alpha\right]$} \\
\hline 4 & {$\left[\cos ^{-1} \frac{d}{2 r_{v}}+\alpha, \alpha+\frac{\pi}{2}\right]$} & {$\left[\pi-a_{5}-\alpha, a_{6}+\pi+\alpha\right]$} \\
\hline 5 & {$\left[\alpha+\frac{\pi}{2}, \pi\right]$} & $\left\lfloor\pi-a_{4}-\alpha, a_{6}+\pi+\alpha\right]$ \\
\hline
\end{tabular}

denoted as $p_{c}(d, n)$ and the probability of event $\bar{C}_{d}^{n}$ is $q_{c}(d, n)$. In addition, $P_{c}(d, n)$ denotes the probability that two nodes can communicate at a distance $d$ through up to $n$ hops.

Adopting the shadowing channel model [11], the signal noise ratio (SNR), $\phi$, in $\mathrm{dB}$ is expressed as

$$
\phi\left(X_{\sigma}, l\right)=P_{t}-P L\left(d_{0}\right)-10 \eta \log _{10}\left(\frac{d}{d_{0}}\right)-X_{\sigma}-P_{n},
$$

where $P_{t}$ is the transmission power, $P L\left(d_{0}\right)$ is the attenuation at the reference distance $d_{0}, X_{\sigma}$ is the shadowing effect random variable and $P_{n}$ is the noise floor.

The symbol error rate, $p_{s}\left(X_{\sigma}, d\right)$ is calculated by

$$
p_{s}\left(X_{\sigma}, d\right)=Q\left(\beta_{2}\left(\phi\left(X_{\sigma}, d\right)-\beta_{1}\right)\right),
$$

where $\beta_{1}$ and $\beta_{2}$ are two parameters obtained by experiments [12]. Note that other error rate models can be used in our scheme. For example, a means to calculate the error rate for MicaZ mote is developed in [10]. The probability that two nodes can communicate in one hop is:

$$
p_{c}(d, 1)=p\left\{C_{d}^{1}\right\}=\int_{-\infty}^{\infty}\left(1-p_{s}(N, d)\right)^{L} f_{X_{\sigma}}\left(X_{\sigma}\right) \mathrm{d} X_{\sigma},
$$

where $L$ is the packet length in symbols, and $f_{X_{\sigma}}\left(X_{\sigma}\right)$ is the pdf of the shadowing effect random variable, $X_{\sigma}$, which is Gaussian.

For the one hop situation, the probability that two nodes can communicate within up to 1 hop is given simply by

$$
P_{c}(d, 1)=p_{c}(d, 1) \text {. }
$$

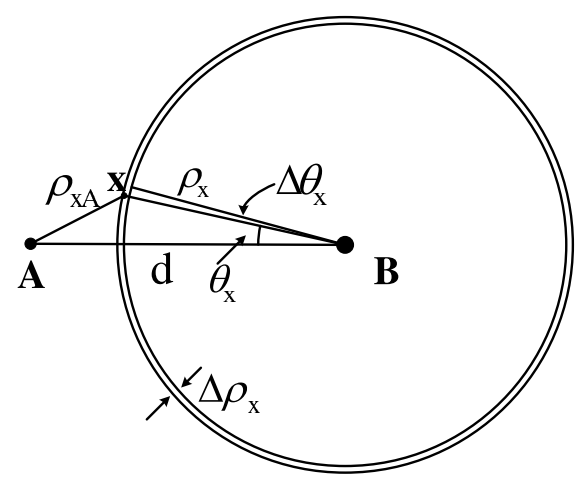

Fig. 2. The multi-hop model.

When the number of hops is greater than 2, we develop our model as shown in Fig. 2. The event that node $A$ has a $n$ hop communication path to node $B$ equals to the event that there is node $x$ at position $\left(\rho_{x}, \theta_{x}\right)$, which has a 1-hop path to $A$ a $(n-1)$-hop communication path to node $B$. Assume the location of the intermediate node, $x$, is $\left(\rho_{x}, \theta_{x}\right)$, where $\rho_{x}$ is the distance between $x$ and $B$, and $\theta_{x}$ is the angle of $x$ with respect to vector $\overrightarrow{B A}$. Thus, the distance between $x$ and $A$ can be expressed as

$$
\rho_{x A}=\sqrt{\left(l-\rho_{x} \cos \theta_{x}\right)^{2}+\left(\rho_{x} \sin \theta_{x}\right)^{2}} .
$$

Given Poisson process density $\lambda$, the probability that at position $\left(\rho_{x}, \theta_{x}\right)$ there is a node is

$$
p_{e}\left(\rho_{x}, \theta_{x}\right)=1-e^{-\lambda \rho_{x} \Delta \rho_{x} \Delta \theta_{x}} \approx \lambda \rho_{x} \Delta \rho_{x} \Delta \theta_{x} .
$$

The approximation holds when the area $\rho_{x} \Delta \rho_{x} \Delta \theta_{x} \rightarrow 0$. The ranges of $\rho_{x}$ and $\theta_{x}$ are $0<\rho_{x} \leq \infty$ and $0 \leq \theta_{x} \leq 2 \pi$.

The probability of 1-hop communication between $x$ and $A$ is $p_{c}\left(\rho_{x A}, 1\right)$, where $\rho_{x A}$ is the distance from $A$ to $x$. Recursively, the probability that node $x$ has a $(n-1)$-hop communication path to node $B$ is $p_{c}\left(\rho_{x}, n-1\right)$. Therefore,

$$
\begin{aligned}
& p_{c}(d, n)=1- \\
& \prod_{\rho_{x}} \prod_{\theta_{x}}\left(1-\left(\lambda \rho_{x} \cdot \Delta \rho_{x} \cdot \Delta \theta_{x}\right) p_{c}\left(\rho_{x A}, 1\right) p_{c}\left(\rho_{x}, n-1\right)\right) .
\end{aligned}
$$

In (15), $1-\left(\lambda \rho_{x} \cdot \Delta \rho_{x} \cdot \Delta \theta_{x}\right) p_{c}\left(\rho_{x A}, 1\right) p_{c}\left(\rho_{x}, n-1\right)$ defines the probability that there is no intermediate node at $\left(\rho_{x}, \theta_{x}\right)$ which satisfies the requirements. Since the relay nodes are deployed independently, the product over $\rho_{x}$ and $\theta_{x}$ is the probability that there is no intermediate node at the whole planar which has 1-hop communication to $A$ and $n-1$ communication to $B$. This is equal to the probability that $A$ and $B$ cannot communicate in $n$ hops. Finally, subtracting that probability from 1 is the probability that the two camera nodes, $A$ and $B$, can have a $n$-hop communication.

Also, the probability that two nodes at distance $l$ can communicate within up to $n$ hops is

$$
\begin{aligned}
P_{c}(d, n)= & p\left\{C_{d}^{1}\right\}+p\left\{\bar{C}_{d}^{1}, C_{d}^{2}\right\}+\cdots \\
& +p\left\{\bar{C}_{d}^{1}, \bar{C}_{d}^{2}, \ldots, \bar{C}_{d}^{(n-1)}, C_{d}^{n}\right\} .
\end{aligned}
$$


The first term in (16) is the probability of communication in 1 hop, the second term is the probability that the two camera nodes cannot communicate in 1 hop but can communicate in 2 hops. Generally, the $n$th term is the probability that the two camera nodes cannot communicate within up to $(n-1)$ hops but can communicate in $n$ hops. The sum is the probability that the two nodes can communicate within up to $n$ hops. Assume that given distance, $d, \bar{C}_{l}^{1}, \ldots, \bar{C}_{d}^{n}$ and $C_{d}^{1}, \ldots, C_{d}^{n}$ are independent ${ }^{1}$, thus, we have

$$
\begin{aligned}
P_{c}(d, n)= & p\left\{C_{d}^{1}\right\}+p\left\{\bar{C}_{d}^{1}\right\} p\left\{C_{d}^{2}\right\}+\cdots \\
& +p\left\{\bar{C}_{d}^{1}\right\} \cdots p\left\{\bar{C}_{d}^{(n-1)}\right\} p\left\{C_{d}^{n}\right\} \\
= & p_{c}(d, 1)+q_{c}(d, 1) p_{c}(d, 2)+\cdots \\
& +\left(\prod_{i=1}^{n-1} q_{c}(d, i)\right) p_{c}(d, n) .
\end{aligned}
$$

\section{Vision Graph Construction}

The communication range of nodes in WSNs is short, hence a camera node's vision graph neighbors may be several hops away. On the other hand, two closely located camera nodes may not be vision graph neighbors. Given a uniformly deployed camera sensor network with relay sensors, we show the probability of vision graph construction as a function of the maximum allowed hop count of the hello messages.

Assume the camera node deployment is a Poisson point process and denote the camera node density as $\kappa$. Given the distance $d$, the probability that a camera node has vision graph neighbors at distance $d$ is

$$
p_{v}(d)=\left(1-e^{-\kappa A_{\mu}}\right) p_{o}(d) \approx \kappa A_{\mu} p_{o}(l),
$$

where $p_{o}(d)$ is the probability that two cameras have an overlapped FOV when the distance is $d$, which is provided in Section II-A, and $A_{\mu}$ is the size of an infinitely small circle area, which is expressed as $A(\mu)=2 \pi d \mathrm{~d} d$.

The paths to some far away vision graph neighbors may not be established because of the limitation of the maximum message hop number. Thus, we consider the probability of constructing the vision graph as the ratio of the connected vision graph neighbors against all vision graph neighbors. Given a camera node $A$, the probability that there are camera nodes at distance $d$ which are vision graph neighbors of $A$ and they can communicate within $n$-hops is

$$
p_{v c}(l, n)=p_{v}(d) P_{c}(d, n),
$$

where $p_{v}(d)$ is given in (18) and $P_{c}(d, n)$ is given in (17).

The probability of constructing the vision graph using $n$-hop communication is

$$
\begin{aligned}
p_{v}(n) & =\frac{\int_{0}^{2 r_{v}} p_{v c}(d, n) \mathrm{d} d}{\int_{0}^{2 r_{v}} p_{v}(d) \mathrm{d} d} \\
& =\frac{\int_{0}^{2 r_{v}} 2 \pi \kappa d p_{o}(d) P_{c}(d, n) \mathrm{d} d}{\int_{0}^{2 r_{v}} 2 \pi \kappa d p_{o}(d) \mathrm{d} d} \\
& =\frac{\int_{0}^{2 r_{v}} p_{o}(d) P_{c}(d, n) d \mathrm{~d} d}{\int_{0}^{2 r_{v}} p_{o}(d) d \mathrm{~d} d},
\end{aligned}
$$

\footnotetext{
${ }^{1}$ In [9], it is pointed out that in reality it is not the case, however, the simulation shows it is a reasonable simplification.
}

TABLE II

PARAMETER LIST.

\begin{tabular}{|c|c|c|c|c|c|}
\hline Parameter & $r_{v}$ & $P_{t}$ & $d_{0}$ & $P L_{d 0}$ & $\eta$ \\
\hline Value & $10 \mathrm{~m}$ & $0 \mathrm{dBm}$ & $1 \mathrm{~m}$ & $55.4 \mathrm{dBm}$ & 4.7 \\
\hline \hline Parameter & $\sigma$ & $P_{n}$ & $\lambda$ & $\beta_{1}$ & $\beta_{2}$ \\
\hline Value & 3.2 & $-95.23 \mathrm{dBm}$ & 0.2 & 2.3851 & 0.9794 \\
\hline
\end{tabular}

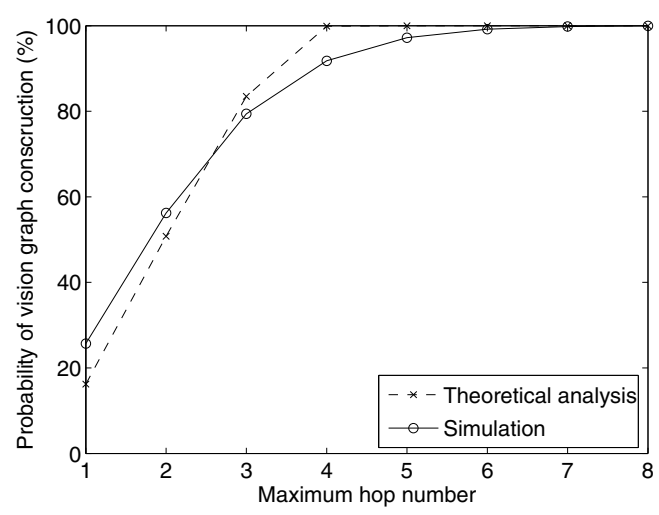

Fig. 3. The comparison of the theoretical analysis and the simulation results for constructing the vision graph.

\section{NUMERICAL ANALYSIS}

In this section, numerical analysis of the system model is performed using Matlab. We first use TOSSIM simulation to verify our model in Section III-A, after which the camera FOV overlap model, the multi-hop communication model as well as the model for constructing vision graph in WMSNs are analyzed in Section III-B. The parameters used in the analysis are listed in Table II.

\section{A. Model Verification}

To verify the models, we have simulated the WMSNs using TOSSIM. In the simulations, 1000 network topologies are generated by a Poisson process, and the size of the field is $50 \mathrm{~m} \times 50 \mathrm{~m}$. In each topology, two cameras nodes are set with random distance and random direction. Unless otherwise noted, he vision angle of each camera is $\frac{5 \pi}{18}$. Note all the camera pairs in the 1000 topologies are vision graph neighbors. Since we consider the ratio of the successful connected vision graph neighbors over all vision graph neighbors, if the two cameras are not vision graph neighbors, they do not have impact on the result. The other parameters are set the same as in Table II.

In Fig. 3, the theoretical and the simulation results of the probability of constructing the vision graph are shown. It is observed from Fig. 3 that the mathematical model captures the characteristics of the FOV overlap and the multi-hop communication. For our settings, instead of unlimited flooding, 5-hop broadcast is sufficient to construct the vision graph with a high confidence (91\%). In other words, when deploying the WMSN, the camera nodes need to broadcast a hello message with maximum hop count of 5 to find the vision graph neighbors.

\section{B. Model Analysis}

In this section, the mathematical models are analyzed. In Fig. 4(a), the probability of camera FOV overlapping over 


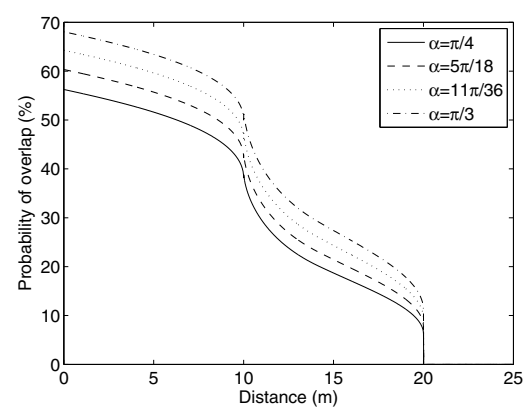

(a)

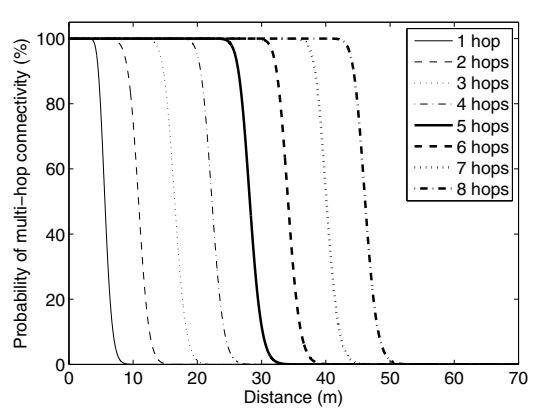

(b)

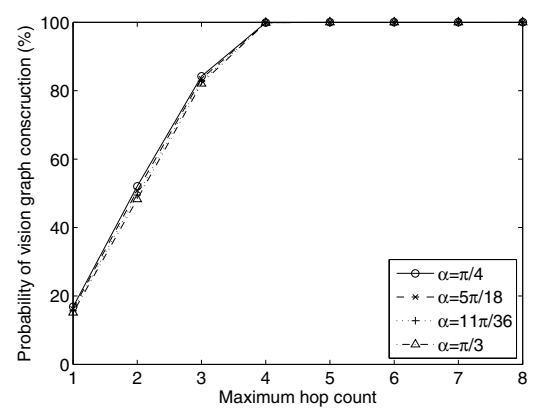

(c)

Fig. 4. (a) The probability of FOV overlap, (b) the probability of multi-hop connectivity and (c) the probability vision graph construction.

distance is shown for different camera vision angles. It is observed that when the camera vision angle is larger, the probability that two cameras have an overlapped FOV is greater. At the distance of $5 \mathrm{~m}$, if the camera vision angle is $\frac{\pi}{3}$, it is $12 \%$ more likely to have a vision graph neighbor than camera vision angle of $\frac{\pi}{4}$. Also, the decrease of the probability over distance is not constant. Around distance of $10 \mathrm{~m}$, which is the vision range, there is a sharp drop. Thus, most vision graph neighbors will be in a close distance.

The probability of connectivity for the multi-hop communication is depicted in Fig. 4(b), where the hop counts are the maximum allowed hop counts. It is shown that if the 1hop coverage is $r$, the coverage for $n$-hop is slightly more than $n r$. For example, at $90 \%$ connectivity, the coverage of 1-hop is $4.57 \mathrm{~m}$, however, the coverage of 3 -hop is $14.73, \mathrm{~m}$ and the overage of 5 -hop is $26.39 \mathrm{~m}$. The reason behind this is that the log-normal channel model does not have a cut-off value as the maximum transmission range. Thus, beyond the $90 \%$ connectivity range, there are still some nodes that receive the message. Accordingly, their transmission can increase the coverage in the next hop. In addition, because the maximum distance of two vision graph neighbors in our setting is $20 \mathrm{~m}$, we would assume the needed hop count is 4 .

The probability of constructing the vision graph as a function of maximum hop counts is shown in Fig. 4(c) for different camera vision angles. It is expected that because when the camera vision angle is greater, the two cameras are more likely to be vision graph neighbors, thus given a maximum hop count, the probability of constructing the vision graph is lower. However, the analysis shows the decrease of the probability is not significant. In fact, when the maximum hop count is 2 , where the difference is most notable, the probability of constructing the vision graph for camera vision angle of $\frac{\pi}{4}$ is $52.08 \%$, and for camera vision angle of $\frac{\pi}{3}$, the probability is $48.28 \%$.

\section{CONClusions}

In this paper, we address the issue of constructing vision graphs in WMSNs. A mathematical model for the camera field of view overlapping is developed to analyze the probability of having vision graph neighbor over distance. Meanwhile, a multi-hop communication model with a realistic channel model is developed to analyze the probability of multi-hop connectiv- ity. Simulations are established to verify these models, which show that the mathematical models capture the characteristics of the FOV overlapping and the multi-hop communication. For our settings, instead of unlimited flooding, 5 -hop broadcast is sufficient to construct the vision graph with high probability.

\section{REFERENCES}

[1] I. F. Akyildiz, T. Melodia, and K. R. Chowdhury, "A survey on wireless multimedia sensor networks," Computer Networks, vol. 51, no. 4, pp. 921 $-960,2007$.

[2] A. Czarlinska and D. Kundur, "Reliable Event-Detection in Wireless Visual Sensor Networks Through Scalar Collaboration and Game-Theoretic Consideration," IEEE Transactions on Multimedia, vol. 10, no. 5, pp. 675690, 2008.

[3] R. Dai and I. F. Akyildiz, "A spatial correlation model for visual information in wireless multimedia sensor networks," IEEE Transactions on Multimedia, vol. 11, no. 6, pp. 1148-1159, 2009.

[4] D. Devarajan, Z. Cheng, and R. Radke, "Calibrating distributed camera networks," Proceedings of the IEEE (Special Issue on Distributed Smart Cameras), vol. 96, no. 10, 2009.

[5] S. Stanislava and H. Wendi, "A Survey of Visual Sensor Networks," Advances in Multimedia, vol. 2009, 2009.

[6] T. Ko and N. Berry, "On scaling distributed low-power wireless image sensors," in Proc. System Sciences, 2006. HICSS'06. Proceedings of the 39th Annual Hawaii International Conference on, vol. 9, 2006.

[7] P. Kulkarni, P. Shenoy, and D. Ganesan, "Approximate initialization of camera sensor networks," Lecture Notes in Computer Science, vol. 4373, p. 67, 2007.

[8] H. Ma and Y. Liu, "Correlation based video processing in video sensor networks," in Proc. IEEE Intl. Conf on Wireless Networks, Communications and Mobile Computing, vol. 2, 2005.

[9] S. Vural and E. Ekici, "Probability distribution of multihop distance in one-dimensional sensor networks," Computer Networks Journal (Elsevier), vol. 51, no. 13, pp. 3727-3749, 2007.

[10] M. C. Vuran and I. F. Akyildiz, "Error control in wireless sensor networks: a cross layer analysis," IEEE/ACM Transactions on Networking (TON), vol. 17, no. 4, pp. 1186-1199, 2009.

[11] M. Zuniga and B. Krishnamachari, "Analyzing the transitional region in low power wireless links" in Proc. IEEE SECON'04, pp. 517-526, Oct. 2004.

[12] http://www.tinyos.net 